\title{
A Case of Aspiration Pneumonia Caused by Gastroesophageal Reflux Disease in A Percutaneous Endoscopic Gastrostomy Patient
}

\author{
Shinji Takahashi ${ }^{1}$ and Naoyuki Kobayashi ${ }^{2}$
}

\begin{abstract}
The patient was an eighty-year-old female. She underwent a Percutaneous Endoscopic Gastrostomy (PEG) in 1999 and has taken her daily nutrition through this until April 2007. She experienced a fever of $39^{\circ} \mathrm{C}$ in May 2007 and was admitted to this hospital for further examination. Computed Tomography (CT) taken at the time of her admission showed pneumonia in the right lung. Furthermore, there was a moderate amount of fluid collected in the esophagus and esophageal dilatation was also observed. The patient eventually died of recurrent aspiration pneumonia. When patients are observed to have fluid collected in the esophagus and esophageal dilatation on CT, then the existence of gastroesophageal reflux disease must be suspected and a careful nutritional strategy must be established in a timely manner. (Kitakanto Med J 2008; $58: 315 \sim 316)$
\end{abstract}

Key Words : aspiration pneumonia, gastroesophageal reflux disease, percutaneous endoscopic gastrostomy

\section{Introduction}

A Percutaneous Endoscopic Gastrostomy (PEG) is widely performed in many medical facilities and gives great benefits for the patients who has difficulties in obtaining sufficient nutrition such as cerebrovascular disorders and neuromuscular diseases which require a long term nutrition supply. Most of these patients are also bedridden. However, gastroesophageal reflux disease remains a problem in such cases because it can cause aspiration pneumonia, which can be fatal in such patients because it is often difficult for them to drain their sputum. This report describes a case of severe aspiration pneumonia due to gastroesophageal reflux disease which occurred after feeding through a PEG.

\section{Case report}

The patient was an eighty-year-old female. She underwent a PEG in 1999, and has since received her nutrition through this until April 2007. During this period, she sometimes suffered from aspiration pneumonia but these symptoms were not critical. She experienced a fever of $39^{\circ} \mathrm{C}$ in May 2007 and was therefore admitted to this hospital for further examina- tion. Computed Tomography (CT) taken at the time of her admission showed pneumonia in the right lung (Fig. 1a). Furthermore, there was also a moderate amount of fluid collected in the esophagus and esophageal dilatation was observed (Fig. 1b). As a result, antibiotics were administered $(3 \mathrm{~g} /$ day SBT/ $\mathrm{CPZ}$ ). The pneumonia gradually improved and feeding was resumed through the PEG three times per day for a few days. Initially, she showed no complications, therefore daily feeding was continued through the PEG. However, she thereafter showed reflux from

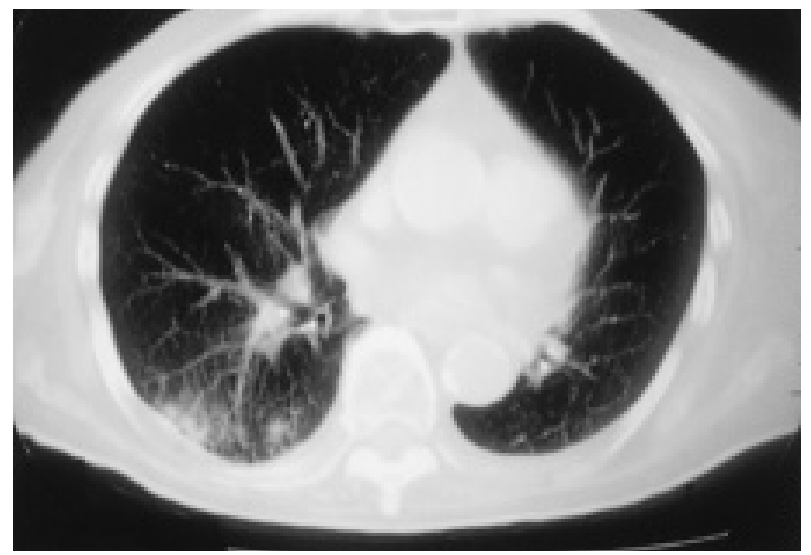

Fig. 1a Computed Tomography taken at the time of her admission showed pneumonia in the right lung.

\footnotetext{
1 Department of Surgery, Kawasaki Saiwai Hospital Nakahara Branch Hospital

2 Department of Surgery, Inagi Municipal

Hospital

Received: May 7, 2008

Address : SHINJI TAKAHASHI Department of Surgery, Kawasaki Saiwai Hospital Nakahara Branch Hospital, 22-1 Kizukisumiyoshicho, Nakaharaku, Kawasaki 211-0021, Japan
} 


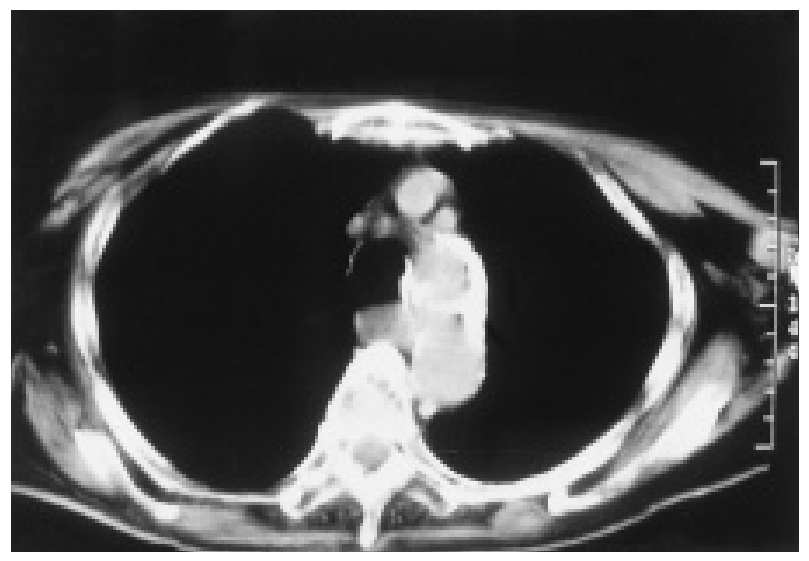

Fig. 1b There was a moderate amount of fluid collected in the esophagus and an esophageal dilatation was observed.

the stomach to the esophagus, and she instantly demonstrated symptoms of aspiration pneumonia again. This disease proved to be extremely resistant to therapy. Sputum cultures showed Pseudomonas aerginosa and MRSA, as a result, $2 \mathrm{~g} /$ day CAZ and $1 \mathrm{~g} /$ day VCM were administered. Furthermore, the gastric juice was passively drained through the PEG to prevent any further gastroesophageal reflux. Despite of these measures, fluid collection in the esophagus still appeared on the CT findings (Fig. 2). The patient eventually died of recurrent aspiration pneumonia in June 2007.

\section{Discussion}

PEG is a very useful procedure when patients have difficulty obtaining sufficient nutrition. Feeding through the gastrointestinal tract is better than total parenteral nutrition (TPN) from a nutritional point of view. It is well known that the rate of sepsis is significantly lower and the morbidity rate in high risk surgical patients is better when patients use PEG in comparison to TPN. ${ }^{1-4}$ Despite these benefits from PEG, we often experience aspiration pneumonia in PEG patients especially in the elderly. The cause of aspiration in a PEG patient is often due to gastroesophageal reflux or aspiration of the patient's own oropharyngeal secretions due to the underlying neurologic deficit or cancer. ${ }^{5}$ Furthermore, it is well-known that the prevalence of hiatus hernia and severe gastroesophageal reflux is higher in the elderly. ${ }^{6,9}$ Increasing age was associated with decreasing abdominal lower esophageal sphincter length and increasing prevalence of ineffective esophageal motility. ${ }^{10}$ As a result, even if patients do not manifest any symptoms of gastroesophageal reflux or aspiration during early period after a PEG was performed, they can suffer from them as they get older, as observed in our case. However, it is difficult to predict the occurrence of gastroesophageal

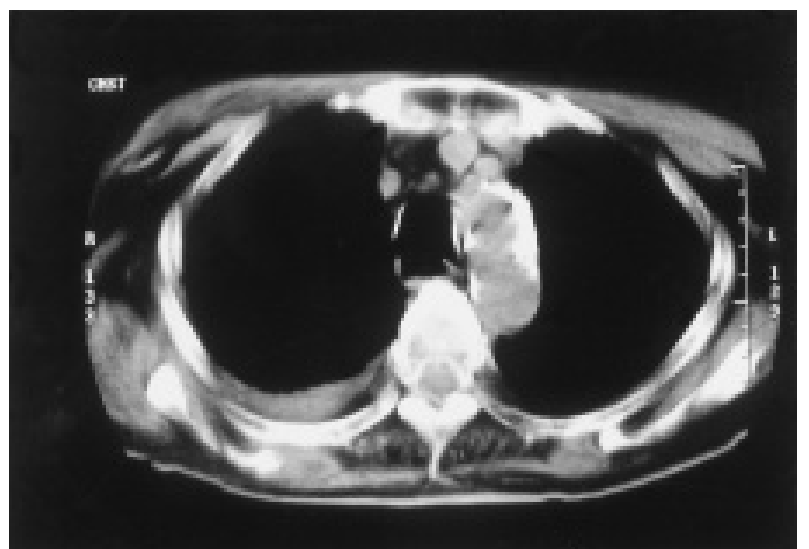

Fig. 2 Fluid collection in the esophagus still appeared on the CT findings.

reflux or aspiration. Thoracic CT findings, such as fluid collection in the esophagus or dilation of esophagus can therefore be a useful marker of gastroesophageal reflux. When patients are observed with such abnormalities on the CT, we should use a highviscosity liquid meal to decrease the occurrence of gastroesophageal reflux. If this is not effective, then we should perform TPN.

\section{References}

1. Moore FA, Moore EE, Jones TN, et al: TEN versus TPN following major abdominal trauma-reduced septic morbidity. J Trauma. 1989; 29: 916-922.

2. Heyland DK, Montalvo M, MacDonald S, et al: Total parenteral nutrition in the surgical patient: a meta-analysis. Can J Surg. 2001 ; 44 : 86-87.

3. Kudsk KA, Croce MA, Fabian TC, et al: Enteral versus parenteral feeding. Effects on septic morbidity after blunt and penetrating abdominal trauma. Ann Surg. 1992; 215 : 503-511.

4. Moore FA, Feliciano DV, Andrassy RJ, et al : Early enteral feeding, compared with parenteral, reduces postoperative septic complications. Ann Surg. 1992; 216: 172-183.

5. Kadakia SC, Sullivan HO: Percutaneous Endoscopic Gastrostomy or Jejunostomy and the Incidence of Aspiration in 79 Patients. Am J Surg. 1992; 164 : 114-118.

6. EI-Serag HB : Time trends of gastroesophageal reflux disease: a systematic review. Clin Gastroenterol Hepatol $2007 ; 5: 17-26$.

7. Zhu H, Pace F, Sangaletti O, et al: Features of symptomatic gastroesophageal reflux in elderly patients. Scand J Gastroenterol 1993; 28 : 235-238.

8. Collen MJ, Abdulian JD, Chen YK: Gastroesophageal reflux disease in the elderly: more severe disease that requires aggressive therapy. Am J Gastroenterol 1995; 90 : 1053-1057.

9. Triadafilopoulos G, Sharma R: Features of symptomatic gastroesophageal reflux disease in elderly patients. Am J Gastroenterol 1997 ; 92 : 2007-2011.

10. LEE J, Anggiansah A, Anggiansah R, et al : Effects of Age on the Gastroesophageal Junction, Esophageal Motility, and Reflux Disease. Clin Gastroenterol Hepatol. 2007 ; 5 : 1392-1398. 